\title{
Towards a New Index for Environmental Sustainability Based on a DALY Weighting Approach
}

\author{
Isabelle Blanc ${ }^{1,2 *}$, Damien Friot ${ }^{1,2}$, Manuele Margni, ${ }^{1,3}$ and Olivier Jolliet ${ }^{1,4}$ \\ ${ }^{1}$ EPFL, Ecole Polytechnique Fédérale de Lausanne, Lausanne, Switzerland \\ ${ }^{2}$ MINES ParisTech, Center for Energy and Processes, Sophia-Antipolis, France \\ ${ }^{3}$ CIRAIG, Ecole Polytechnique de Montréal, Canada \\ ${ }^{4}$ Environmental Health Sciences, School of Public Health, University of Michigan, USA
}

\begin{abstract}
Composite indicators are synthetic indices that are used to rank country performances in specific policy areas. Many do, however, suffer from methodological difficulties. Specific difficulties linked to indices for environmental sustainability are analyzed through the illustration of several sets. The most critical issues are linked with a poor analytical framework and a lack of common unit for the aggregation. Some measure directly the state of the environment while other use proxies such as pressure or response indicators or even a mix of these. A new composite index for environmental sustainability was developed in the EU project EPSILON, which aimed at assessing European regional sustainability for policy decision making related to the improvement of regional sustainability. Indicators are expressed according to a coherent framework issuing from the 'driving force-pressure-state-impactresponse' approach with an innovative weighting scheme derived from human health impact assessment based on disability adjusted life years (DALYs). Results are compared with a more conventional aggregation technique based on an equal weighting coupled to various normalization techniques. Copyright (C) 2008 John Wiley \& Sons, Ltd and ERP Environment.
\end{abstract}

Received 25 February 2005; revised 6 March 2008; accepted 10 March 2008

Keywords: environmental sustainability; composite index; DALY weighting; human health; regional benchmarking; impact assessment

\section{Introduction}

OMPOSITE INDICATORS ARE SYNTHETIC INDICES THAT ARE USEFUL IN RANKING COUNTRIES' PERFORMANCES AND FOR setting policy objectives. Existing indices cover a wide range of fields such as economics, environment, globalization or even society and innovation. A complete and systematic list reporting these indices is to be found on the website of the European Commission Joint Research Center on Composite Indicators. ${ }^{\mathrm{I}}$

\footnotetext{
* Correspondence to: Isabelle Blanc, Ecole des Mines de Paris, Process and Energy Center, BP 207, F-06904 Sophia-Antipolis, France. E-mail: isabelle.blanc@ensmp.fr

${ }^{\mathrm{I}} \mathrm{http}: / /$ farmweb.jrc.cec.eu.int/ci/Indexes.htm.
} 
Examples of composite indicators focusing on the environment are numerous and include the Environmental Sustainability Index (ESI, 2005), the ecological footprint (WWF, 2004) and the ISSI (Ronchi et al., 2002). Their scopes are diverse and the resulting assessments highly dependent on the underlying frameworks, since the development of aggregate environmental indices has not reached a universal consensus. Methodological improvement is necessary first to clarify explicitly all underlying assumptions and second to take advantage of the latest innovative research related to impact assessment.

Key methodological difficulties encountered with the elaboration of composite indices are analyzed through the illustration of several sets of indices related to environmental issues. Lessons learned from these investigations have been integrated in the development of a new environmental index for which methodological choices are presented in this study.

The new environmental sustainability index was developed within the EPSILON ${ }^{2}$ project (Bonazountas et al., 2004; Blanc et al., 2004, 2005b). The project aimed at assessing European regional sustainability in order to provide a tool for policy decision making related to the improvement of regional sustainability. EPSILON delivered a GIS integrated computerized model for benchmarking regions at EU-wide level through an aggregation of indicators represented on sustainability maps at NUTS 3 II and then at NUTS III level (Blanc et al., 2005a). Sustainability indicators have been defined along four pillars: the environmental, economic, social and institutional dimensions (UN-CSD, 200I). The key fundamentals of the new environmental sustainability are a coherent structure, which relies on the analytical DPSIR framework (driving forces-pressure-state-impact-response) from the European Environmental Agency (EEA, 2003), coupled to an innovative weighting scheme based on human health impact assessment.

\section{Composite Indices: Key Methodological Issues}

Although composite indices are commonly provided as tools for ranking and benchmarking (OECD, 2004), their elaboration is problematic. The most critical issues are related first to the choice of an analytical framework and second to the full implementation of its logic. While the choice of a poor analytical framework results in a distortion between the aim of the assessment and what is effectively measured, a partial implementation can cause double counting.

\section{An Analytical Framework to Avoid Inconsistencies}

A sound analytical framework helps in differentiating and categorizing indicators to avoid inconsistencies. Within the environmental assessment field, the DPSIR approach initiated by EEA provides such a basis. Most existing sets of indicators are therefore based on, or at least refer to, the DPSIR approach (Berger-Schmitt and Noll, 2000). This model is based on the OECD's PSR model, originally developed as a conceptual framework of a system of environmental indicators. It differentiates categories of indicators in order to explain the modification of the state of the environment resulting from the pressure imposed by human activities on the environment and the impacts of such changes. It also includes the individual or collective response to these impacts.

Several sets of indicators have been constructed and organized in the DPSIR logic, e.g. the Environmental Sustainability Index (ESI, 2005), the 2006 Pilot Environmental Performance Index (EPI, 2006) and an Italian regional index (Clerici et al., 2004). Three types of weakness can be identified within the existing sets: the lack of coherence in the choice of categories and indicators, the lack of a common unit for aggregation and the missing impact level.

\footnotetext{
${ }^{2}$ EPSILON is an EU RTD-IST project - EC Contract IST-200I-32389. Partners: EPSILON-NTUA, Greece; PbS, Germany; EPFL, Switzerland; University of Minho, Portugal, and Mice, Italy.

${ }^{3}$ NUTS, Nomenclature of Territorial Units for Statistics, is a standard geo-code for referencing the administrative division of countries for statistical purposes.
} 


\section{Aggregating Indicators and Categories of Different Levels}

Environmental performance is generally measured using indicators that are distributed over different categories. Two important sources of errors should be recognized. First, although indicators may be adequately attributed to categories, the choice of the level of assessment is not systematic at the category level. For example, in the earlier version of the Pilot Environmental Performance Index (EPI, 2002) all indicators defining the air quality correspond to the state level $\left(\mathrm{NO}_{2}, \mathrm{SO}_{2}\right.$, total suspended particulates, and lead concentrations) but the indicators defining the land protection correspond to the response level (share of glass or paper recycled, waste disposal in landfills per populated land area, and protected areas as share of total area).

Second, indicators of different levels are often mixed within the same category. In the set of indicators developed by Clerici et al. (2004), the category 'water quality' mixes a state indicator (nitrate concentration), a response indicator (fraction of the population served by secondary and tertiary treatment) and a pressure indicator (tons of pigs and cattle per $\mathrm{km}^{2}$ ). After aggregation it is not clear what is measured: the quality of the environment or the quality of the policies put in place to improve the environmental conditions.

Both type of error lead to ambiguous interpretations and to a risk of double-counting, e.g. measuring once the human action and then its result in terms of environmental quality. Furthermore, using response indicators as proxies for state indicators does not allow for a parallel assessment of both the policies and their goals.

The Environmental Sustainability Index (ESI, 2002) avoids the problems and is structured according to several categories that are fully in line with the DPSIR structure. A later version of this index (ESI, 2005) has extended the number of indicators per category but kept the same coherence related to the DPSIR framework. This shows that it is possible to clearly differentiate between actions/policies and the environmental conditions in the construction of indicators.

\section{Lack of a Common Unit for Aggregation}

A common reference between indicators is crucial for obtaining an aggregate with a clear meaning, i.e. not a disparate combination, and an unbiased estimation. This reference can be either theoretical, i.e. choosing indicators assumed conceptually equivalent or statistical, using techniques of normalization. Several indicator sets suffer from the inconsistency caused by the mixing of indicators without a common unit or known equivalency between them. In this situation, the resulting aggregated indicator is biased since it is influenced both by the explicit weights and by the range of each of the indicator.

\section{The Missing Impact Level}

None of the examined sets (ESI, 2005; EPI, 2006; Clerici et al., 2004) use the DPSIR framework fully: there are no indicators at the impact level. This level is, however, essential when one aims at assessing environmental sustainability since it is the only one taking into account the resulting effects of an emission. The same emission (P), for example, will not have the same consequence (I) if it is deposited in a densely populated area or in a desert region. Models of impact assessment, e.g. Impact2002+ (Jolliet et al., 2003), have, however, been developed over the last few years, providing this link between a pollutant emission, its transport within environmental media and the actual damages to ecosystems or human health.

\section{The Effect of Standardization Techniques and Weighting on Composite Indices}

\section{Steps for a Composite Indicator}

Aggregating a collection of indicators in a coherent structure is a major challenge. In order to overcome some of the pitfalls encountered when elaborating a composite indicator several methodological frameworks have been presented (Booysen, 2002; Salzman, 2003; Nardo et al., 2005; Saisana et al., 2002). Composite indices are usually based on additive techniques with equally weighted indicators. Although these techniques are objective as such, 
some subjectivity is nevertheless introduced in composite indices (Booysen, 2002). A full understanding of the techniques is necessary to appreciate what is really addressed in a composite index.

There are four steps in constructing a composite index:

(I) the selection,

(2) the scaling,

(3) the weighting and

(4) the aggregation of the variables.

In the following, we consider issues related to scaling and weighting and present a practical illustration by applying the method to the aggregation of various pollutants.

\section{Description of Scaling Methods}

Scaling techniques aim at transforming (normalizing) variables to make them comparable on the basis of a common unit. The most commonly used techniques for indicator sets are the $Z$-score, the distance from the leader, the distance from the mean and the min-max.

The $Z$-score represents the deviation of a value from the mean divided by the average deviation from the mean of the variable. It standardizes variables so that their mean is zero and standard deviation unity. Positive and negative values are possible. The distance from the group leader assigns Io०\% to the leading value and the other values are ranked as percentage points away from the leader. The distance from the mean assigns the mean value to I00\% and the other values are scored according to their distance from the mean. The LST method (distance from the best and the worst performers), also called the min-max method, scales the values between the best and the worse performance. Each element is compared with the best one and its relative position between the extremes is assessed.

The four methods result in a unitless indicator. The first three do not, however, provide a normalized range: while values are normalized, the distance between the normalized minimum and maximum values varies with the dataset. This is not an issue when one considers only one indicator, since the standardization of a single variable does not change the ranking between indicator values (Freudenberg, 2003). It becomes, however, an issue in the case of a composite indicator, which is based first on a scaling and then on a weighting, since these different ranges act as an implicit weighting scheme. The $Z$-score and the distance from the mean have the advantage of not being too sensitive to extreme values, whereas the distance from the group leader and the min-max are directly dependent on extreme values that may only reflect errors in input data.

\section{Description of Weighting Methods}

Composite indicators always require weighting of indicators, which means establishing a ranking among the different indicators. Such ranking can be a delicate task as indicators are addressing different issues that may not be related.

There are several ways to weight indicators:

- expert weighting based on scientific expertise;

- expert weighting based on societal determination (policy makers or social surveys);

- weighting based on statistical data treatment such as principal component analysis.

The weighting step is often avoided on the pretext that values are involved and that the best estimate is an equal weighting. The preceding standardization step provides therefore the numerical counterpart of the conceptual equivalence. This is acceptable when ranges are normalized, as in the min-max method: the difference in the real world between the worst and the best score per indicator is perceived to be equally important and so is the range. This is, however, inadequate with other normalization techniques since this matching is not provided and a bias results from the implicit weighting scheme conveyed by the varying ranges.

Skipping any weighting step after standardizing the data ranges is therefore not equivalent, from a numerical point view, to an implicit equal weighting. 


\section{Application of Scaling Methods with Equal Weighting}

To illustrate the different approaches, the four presented methods are applied to a common case study, creating a composite index for air pollution due to

(I) annual concentrations of particulates matter (PM);

(2) annual concentrations of heavy metals (HM: lead, cadmium and mercury);

(3) annual concentrations of persistent organic pollutants (POPs 4 : PCB, BaP, HCB and PCDD/Fs).

Figure I presents the observed concentrations for these three classes of emissions.

The approach is illustrated in detail for the min-max standardization with an implicit equal weighting strategy. The normalized indicators are in this case equivalent both from a numerical and from a conceptual perspective, although their absolute values are very different (Table I).

Heavy metals account for around a third of the normalized index and POPs contribute between $\circ$ and $40 \%$ (Figure 2). These results are typical for existing environmental indices: there is no similarity between countries and each indicator makes a significant contribution to the final index.

Figure 3 presents the results of the four scaling methods with an implicit equal weighting scheme. Some differences are noticeable between the scaling methods: six countries out of I5 differ by more than two rank positions. Portugal shows the highest discrepancy, with a sixth rank for the min-max method and a I2th position for the $Z$-score, followed by Luxembourg, with an eighth rank for the Z-score and a I3th rank for the 'distance from the average' method. This shows that an indicator with extreme values will have intrinsically greater effect on the composite indicator and will reward exceptional behavior when one uses, for example, the $Z$-score method (Nardo et al., 2005). This is the case for Luxembourg: extremely good results for the PM indicator are rewarded with a favorable eighth rank compared with the 13 th rank given by the 'distance from the average' method.

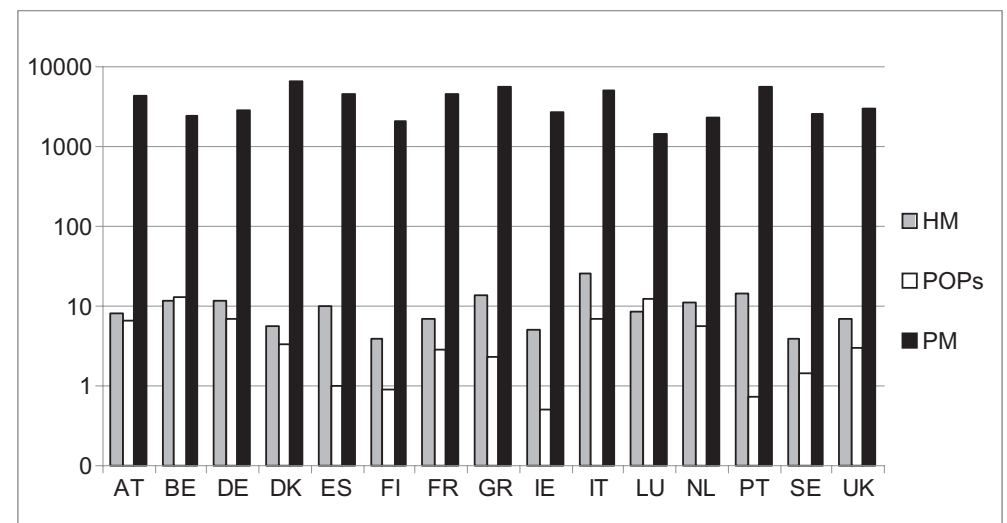

Figure 1. PM, POP and heavy metal concentrations in $\mathrm{ng} / \mathrm{m}^{3}$ (log scale) issued from EMEP (2003) data (year 2000)

\begin{tabular}{llll}
\hline & Heavy metals & POPs & PM \\
\hline Min. value $\left(\mathrm{ng} / \mathrm{m}^{3}\right)$ & $\mathrm{I} .30$ & 0.13 & $\mathrm{I} 439.98$ \\
Max. value (ng/m $\left./ \mathrm{m}^{3}\right)$ & 8.50 & 3.17 & $65 \mathrm{I} 4.00$ \\
Delta (max. - min.) $\left(\mathrm{ng} / \mathrm{m}^{3}\right)$ & $7.2 \mathrm{I}$ & 3.05 & 5074.02 \\
\hline
\end{tabular}

Table 1. Minimum and maximum values on cumulated concentrations of heavy metals, persistent organic pollutants and particulate matter over all countries (EMEP data 2003 over 15 EU countries)

\footnotetext{
${ }^{4}$ Persistent organic pollutants (POPs) are chemical substances that persist in the environment, bio-accumulate through the food web and pose a risk of causing adverse effects to human population and the environment.
} 


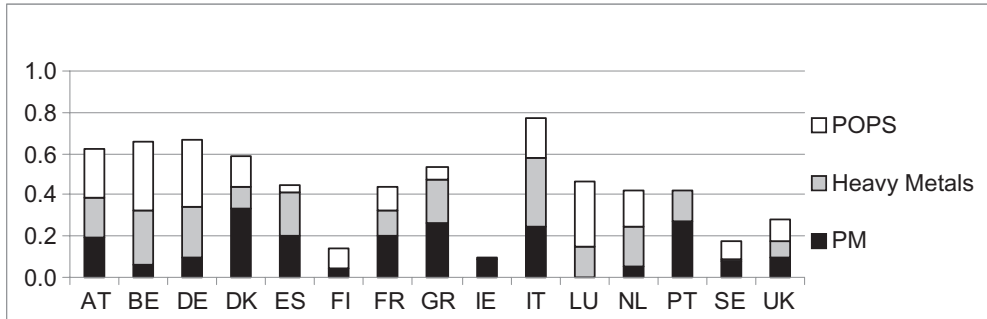

Figure 2. Benchmarking $15 \mathrm{EU}$ countries at present level of emissions with normalized index based on min-max and equal weighting scheme

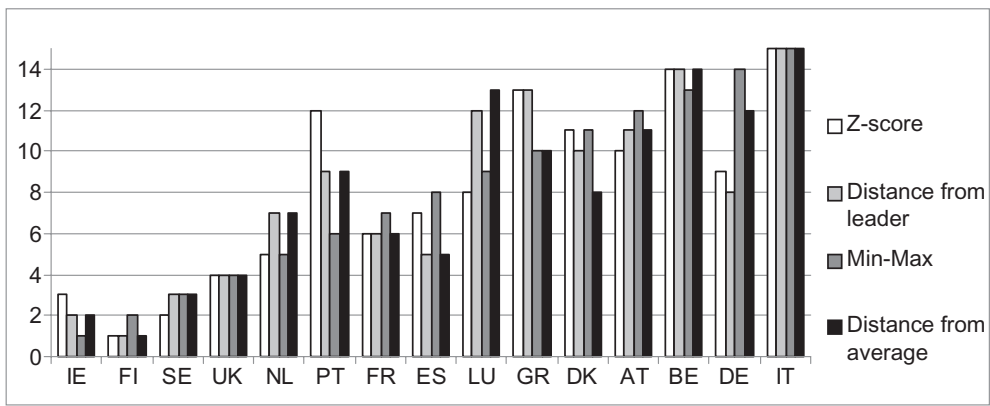

Figure 3. Relative ranking among the 15 EU countries: for the four different normalization techniques and equal weighting

The dependence of the normalization techniques on the datasets is an inherent weakness of composite indicators. To overcome this dependence, a solution is to skip this step by using an elaborated weighting scheme. This is what is proposed in EPSILON and the DALY-based approach.

\section{The EPSILON Approach for an Air Index}

\section{EPSILON: a New Set of Indicators for Assessing Environmental Sustainability}

The EPSILON set of indicators has been developed to meet the requirements for a coherent theoretical framework and for the choice of relevant levels for the categories and indicators. Sustainability is addressed over four dimensions/pillars: the environmental, the economic, the social and the institutional dimensions. Four separate indices have been elaborated, one for each sustainability dimension. Within each pillar, prior to any weighting, great attention has been given to the model coherence through the definition of relevant indicators.

To respect the DPSIR framework, the EPSILON structure has been designed to (Blanc et al., 2005b)

(I) assess the state of the environment with state indicators (and avoid any mixing with response indicators or pressure indicators);

(2) consider the impact level with an environmental weighting scheme based on damages;

(3) combine environmental indicators based on a common unit at impact level;

(4) complete the environmental state with a separate collection and grouping of possible responses at national or regional level.

As a result, the environmental pillar (Table 2) is defined as a structure of four themes, each theme being defined with sub-themes (Blanc et al., 2005a). The air theme illustrates the approach (Figure 4). 


\begin{tabular}{ll}
\hline Theme index & Sub-theme index \\
\hline Air index & Climate change \\
& Air quality I \\
& Air quality 2 \\
& Noise \\
Soil index & Soil degradation \\
& Soil toxicity \\
& Soil loss \\
Water index & Water quantity \\
& Surface water quality \\
& Ground water quality \\
& Ocean quality \\
Land index & Fragmentation \\
& Naturalness \\
& Wilderness \\
& Wetlands \\
\end{tabular}

Table 2. Structure of the environmental pillar within EPSILON

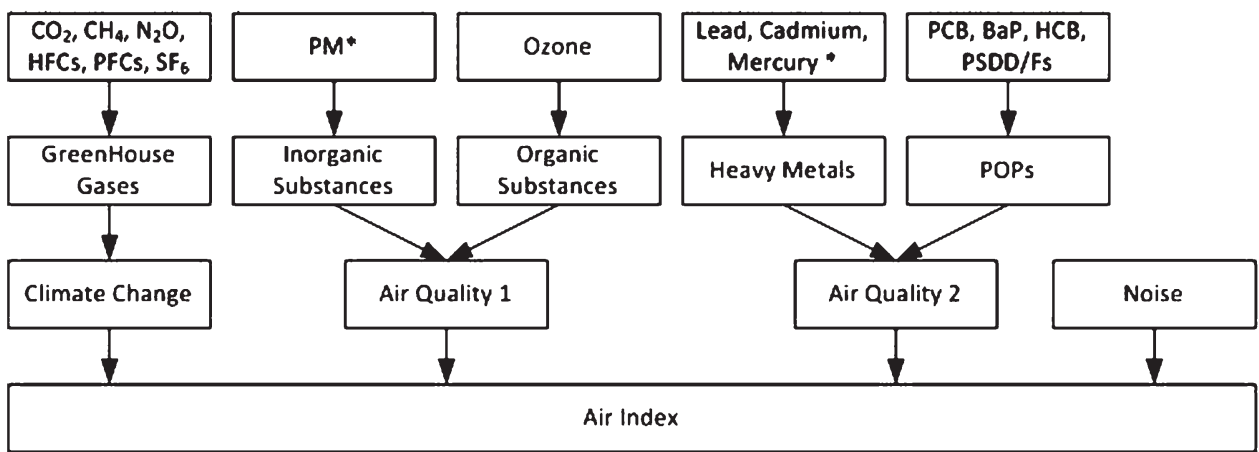

(a)

(b)

(c)

(d)

Figure 4. Air index of the EPSILON environmental index: (a) sub-indicators; (b) indicators; (c) sub-theme; (d) theme

\section{A New Weighting Technique Based on DALY Weights Within the EPSILON Composite Indicator}

Within EPSILON, the DPSIR is fully utilized by using the impact category to aggregate environmental state indicators. The weighting scheme is based on the assessment of the potential impacts on human health. Common units are DALYs (disability adjusted life years), which are calculated with the impact assessment multi-media model IMPACT 2002+ (Jolliet et al., 2003; Pennington et al., 2005), tracing toxics from their emissions to their assimilation by human bodies (Murray and Lopez, I996; Crettaz et al., 2002; Pennington et al., 2002). The weightings corresponding to the eight indicators that form the air index are given in Figure 5.

Weighting coefficients (expressed in DALY/person/concentration) are applied to each pollutant concentration to provide a global assessment of DALY/person for the air index within each country. No standardization step is required in this case.

Benchmarking I5 EU countries applying this DALY weighting is shown in Figure 6. The picture is completely different from the equal weighting. Results range from 0.I7 days lost/person/year for Luxembourg to 0.54 days lost/person/year for Denmark. Heavy metals are not visible. Their low contribution to the annual days lost per 


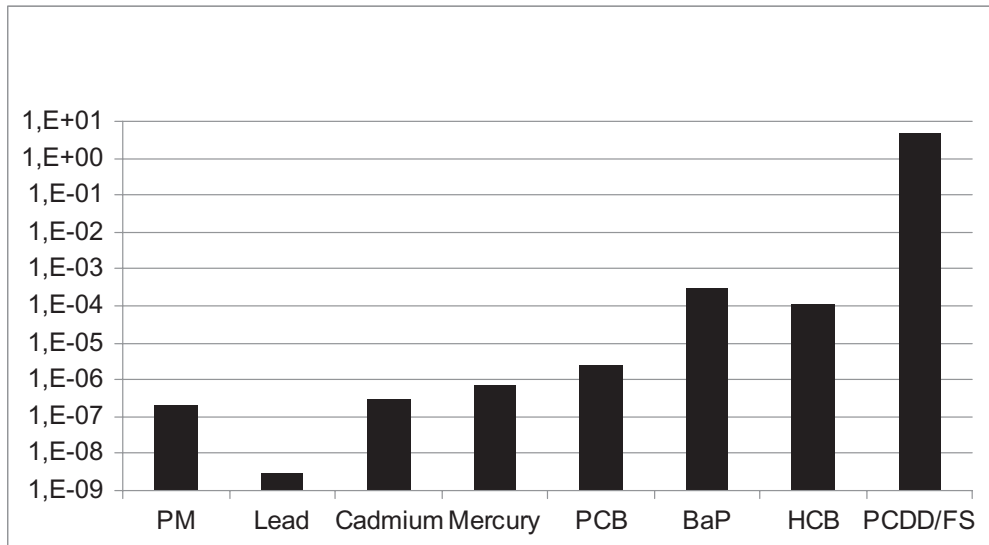

Figure 5. Weighting coefficient characterizing impacts on human health per unit concentration of the considered emissions, in $\mathrm{DALY} / \mathrm{pers} / \mathrm{ng} / \mathrm{m}^{3}$ issued from IMPACT $2002+$

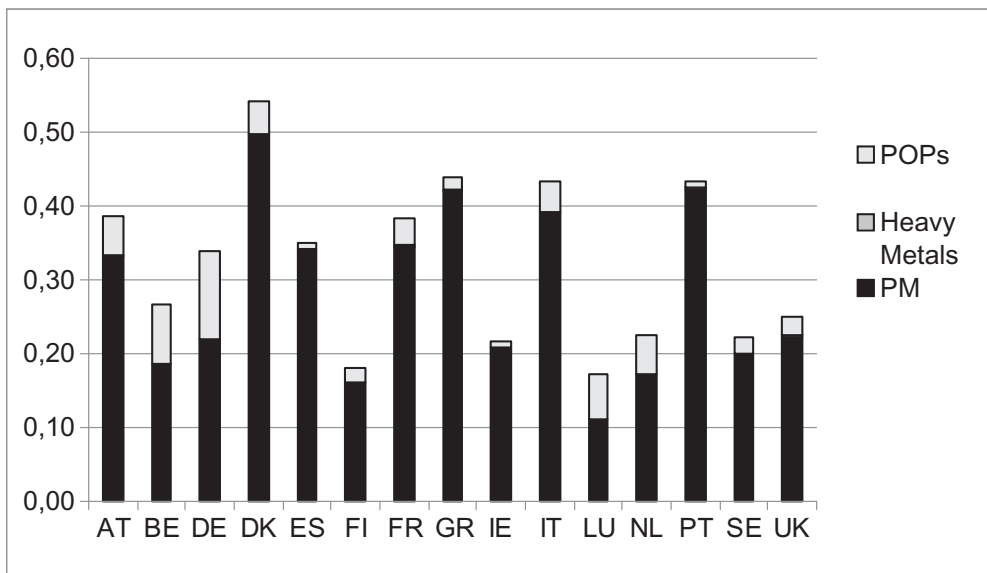

Figure 6. Benchmarking $15 \mathrm{EU}$ countries at present levels of emissions with the DALY weighting scheme (in days lost/person/ year)

person is caused both by the low weights attributed to heavy metals due to their low impact on human health compared with POPs and PM and by the absolute low values of HM concentrations.

Most of the days lost are related to PM concentrations (over 90\% for Io countries out of I5) in European countries, but in some countries POP concentrations are not negligible (Belgium, Germany, Luxembourg and the Netherlands, where they represent between 20 and $30 \%$ of the total days lost). This is in strong opposition to what an equal weighting, as shown earlier, would suggest. The knowledge of the potential impact on human health guides therefore the proper selection of relevant indicators, by focussing on the main ones.

\section{Comparing the EPSILON and the Min-Max Approach with Equal Weighting}

This section compares the EPSILON air composite based on DALYs (Figure 6) with the min-max standardization with an implicit equal weighting strategy (Figure 2). This is the best default strategy when no scientific knowledge is available for weighting.

The two approaches lead to large differences in ranking for several countries (Figure 7). Thus Luxembourg moves from a fairly poor performance (Rank 9) when applying the equal weighting to standardized values to the best one (corresponding to the lowest air concentrations) with the DALY weighting scheme. Heavy metal and POP 


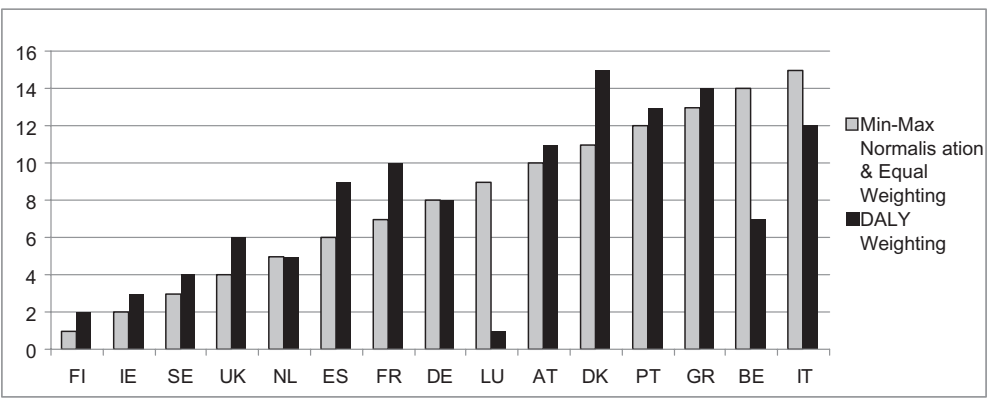

Figure 7. Relative ranking among the 15 EU countries: comparison of the DALY weighting and the normalized min-max values with equal weighting

concentrations are reported nearly at the highest level within the I5 EU range while PM concentrations are the lowest of the I5 EU countries (see Figure I, with $\mathrm{I} 440 \mathrm{ng} / \mathrm{m}^{3}$ for Luxembourg). Normalizing the three data ranges means POP and HM concentrations are given an equal weight when a LST technique is used. This maximizes the influence of the highest score for POPs. With the DALY weighting technique the importance of the high POP value is minimized due to a fairly low DALY factor.

Belgium (BE) and Germany (DE) also show large differences in ranking depending on the technique used. Both countries have fairly high values for POP concentration. With the min-max method and equal weighting the air index is overestimated. With the DALY weighting the POPs have a smaller impact on the index and the rank is improved. In contrast, the ranks of Portugal and Greece worsen when the DALY weighting is used. These countries have low POP concentrations but relatively high PM concentrations (corresponding to high DALY factor).

\section{Discussion and Conclusions}

After recognizing the importance of different standardization techniques for composite indices, it has been underlined how highly sensitive they are to underlying datasets. The choice of a weighting scheme is also a delicate step and a scientific weighting for composite environmental indicators differs greatly from an equal weighting applied to a standardized data range. The differences can be explained and they show that necessary scientific expertise is required to set a proper weighting scheme. The suggested composite environmental index relies on a scientific scheme that makes the most of the DPSIR by linking the environmental state to impacts on human health.

Based on the analysis and the results, the following recommendations can be made for to the design of a composite sustainability index.

I. Choose to rank indicators first based on available scientific impact modeling, such as the weighting established for the greenhouses gases (IPCC, 200I), or a weighting scheme based on life cycle impact assessment, e.g. the DALY factors for the environmental sustainability index.

2. Adopt otherwise the implicit equal weighting approach with a normalization technique providing equal ranges such as the linear scaling technique. Such an approach avoids specific ranking between indicators but requires a clever selection of equivalent indicators. As illustrated here, such an approach is not recommended for an environmental sustainability index but could be applied with the other dimensions of sustainability (economic, social or institutional), where the assumption of equivalence between indicators may be less problematic.

Further extensions are necessary for the new DALY weighting scheme if one aims at a fully coherent index for environmental sustainability:

- DALY weighting coefficients should be derived for a large number of indicators covering all media (soil, water) to ensure a full compatibility for the weighting scheme;

- better knowledge of dose response functions should be integrated in the estimation of DALYs;

- damage assessment should not be limited to human health but should also consider ecosystems. 


\section{References}

Berger-Schmitt R, Noll H-H. 2000. Conceptual Framework and Structure of a European System of Social Indicators, EU-Reporting Working Paper 9, subproject 'European System of Social Indicators'. Centre for Survey Research and Methodology (ZUMA) Social Indicators Department: Mannheim.

Blanc I, Friot D, Margni M, Jolliet O. 2004. How to assess the environmental state of EU regions with the global concept of sustainability? In 18th International Conference on Informatics for Environmental Protection - Enviroinfo Geneva Conference, 2004. www.enviroinfo2004.org/ cdrom/datas/BlancI.htm [4 March 2008].

Blanc I, Friot D, Margni M, Jolliet O. 2005a. Assessing environmental sustainability within the EPSILON project: first version of an innovative approach. In Third Dubrovnik Conference on Sustainable Development of Energy, Water and Environment, 2005. Faculty of Mechanical Engineering and Naval Architecture, University of Zagreb: Zagreb.

Blanc I, Friot D, Margni M, Jolliet O. 2005b. WP3 Documentation, Part 1 The Sustainability Model Framework and Indicators, EPSILON working document.

Bonazountas M, Smirlis Y, Kallidromitou D. 2004. Assessing sustainability of EU regions: the case of the EPSILON tool. In 18 th International Conference on Informatics for Environmental Protection - Enviroinfo Geneva Conference, 2004. www.enviroinfo2004.org/cdrom/datas/asses sing\%20sustainability.htm [4 March 2008].

Booysen F. 2002. An overview and evaluation of composite indices of development. Social Indicators Research 59: II5-I5I.

Clerici N, Bodini A, Ferrarini A. 2004. Sustainability at the local scale: defining highly aggregated indices for assessing environmental performances. The province of Reggio Emilia (Italy) as a case study. Environmental Management 34(4): 590-608.

Crettaz P, Rhomberg L, Brand K, Pennington DW, Jolliet O. 2002. Assessing human health response in life cycle assessment using EDios and DALYs: carcinogenic effects. International Journal of Risk Analysis 22: 929-944.

EMEP. 2003. Transboundary Pollution by HM and POPs, MSC-E Note 6/2003. Meteorological Synthesizing Centre - East: EMEP Cooperative Programmme for Monitoring and Evaluation of the Long-Range Transmission of Air Pollutants in Europe.

Environmental Performance Index (EPI). 2002. Pilot Environmental Performance Index, an initiative of the Global Leaders of Tomorrow Task Force, World Economic Forum, annual meeting 2002 - Yale University-Columbia University. http://sedac.ciesin.columbia.edu/es/esi/ archive.html [4 March 2008].

Environmental Performance Index (EPI). 2006. Pilot 2006 Environmental Performance Index. Yale Centre for Environmental Law and PolicyCIESIN Columbia University. http://sedac.ciesin.columbia.edu/es/epi/2006archive.html [4 March 2008].

Environmental Sustainability Index (ESI). 2002. Environmental Sustainability Index, Benchmarking National Environmental Stewardship, an Initiative of the Global Leaders of Tomorrow Task Force, World Economic Forum, annual meeting 2002 - Yale University-Columbia University. http://sedac.ciesin.columbia.edu/es/esi/archive.html [4 March 2008].

Environmental Sustainability Index (ESI). 2005. Environmental Sustainability Index, Benchmarking National Environmental Stewardship. Yale University-Columbia University. http://sedac.ciesin.columbia.edu/es/esi/downloads.html [4 March 2008].

European Environment Agency (EEA). 2003. Europe's Environment: the Third Assessment, EEA: Copenhagen-Office for Official Publications of the European Communities: Luxembourg.

Freudenberg M. 2003. Composite Indicators of Country Performance: a Critical Assessment, OECD Science, Technology and Industry Working Paper 2003/I6, OECD Directorate for Science, Technology and Industry.

Intergovernmental Panel on Climate Change (IPCC). 200I. Climate Change 2001: the Scientific Basis. IPCC-Cambridge University Press: Cambridge.

Jolliet O, Margni M, Humbert S, Payet J, Rebitzer G, Rosenbaum R. 2003. IMPACT 2002+: a new life cycle impact assessment methodology. International Journal of Life Cycle Assessment 8: 324-330.

Murray C, Lopez A. I996. The Global Burden of Disease, a Comprehensive Assessment of Mortality and Disability from Diseases, Injuries and Risk Factors in 1990 and Projected to 2020, Global Burden of Disease and Injury Series Vols I, 2. Harvard School of Public Health-World Health Organization-World Bank: Cambridge, MA.

Nardo M, Saisana, M, Saltelli, A, Tarantola S. 2005. Tools for Composite Indicator Building, EUR 2I682 EN. European Commission - Applied Statistics Group - JRC: Ispra, Italy.

OECD. 2004. Workshop on Composite Indicators of Country Performance, 2004. http://www.oecd.org/document/o/o,3343,fr_2649-37443_ 29393984_I_I_I_37443,00.html [4 March 2008].

Pennington DW, Crettaz P, Tauxe A, Rhomberg L, Brand K, Jolliet O. 2002. Assessing human health response in life cycle assessment using EDios and DALYs: non-carcinogenic effects. International Journal of Risk Analysis 22: 945-96I.

Pennington DW, Margni M, Amman C, Jolliet O. 2005. Multimedia fate and human intake modeling: spatial versus non-spatial insights for chemical emissions in Western Europe. Environmental Science and Technology 39: III9-II28.

Ronchi E, Federico A, Musmeci F. 2002. A system oriented integrated indicator for sustainable development in Italy. Ecological Indicators 2: I97-2I0.

Saisana M, Tarentula S, Saltelli A. 2002. State-of-the-Art Report on Current Methodologies and Practices for Composite Indicator Development, EUR 20408 EN. European Commission - Applied Statistics Group - JRC: Ispra, Italy.

Salzman J. 2003. Methodological Choices Encountered in the Construction of Composite Indices of Economics and Social Well-Being. Centre for the Study of Living Standards Report 2004-05. CSLS.

UN Commission on Sustainable Development (UN-CSD). 200I. Indicators of Sustainable Development Framework and Methodologies. UN-CSD: New York.

World Wide Fund For Nature (WWF). 2004. The Living Planet Report. WWF: Gland, Switzerland. 